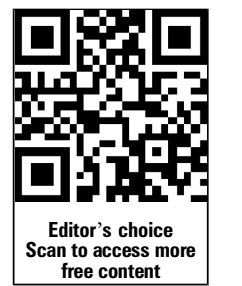

${ }^{1}$ Swiss EoE Clinic, Department of Gastroenterology and Hepatology, University Hospital Basel, Basel, Switzerland ${ }^{2}$ Department of Gastroenterology and Hepatology, Centre Hospitalier Universitaire Vaudois/CHUV, Lausanne, Switzerland

Correspondence to Professor Alex Straumann, Swiss EoE Clinic and EoE Research Network, Roemerstrasse 7, Olten 4600, Switzerland; alex.straumann@hin.ch

Received 4 February 2014 Revised 20 February 2014 Accepted 21 February 2014 Published Online First 3 April 2014

\title{
Update on basic and clinical aspects of eosinophilic oesophagitis
}

\author{
Alex Straumann, ${ }^{1}$ Alain Schoepfer ${ }^{2}$
}

\section{ABSTRACT}

The identification of a distinct syndrome, designated eosinophilic oesophagitis (EoE), with its own clinical and histopathological characteristics, was first described in the early 1990s. Meanwhile intense research has uncovered many molecular, immunological and clinical aspects of this chronic-inflammatory disorder. This article focuses exclusively on basic and clinical insights of EoE gathered during the last few years. Regarding aetiopathogenesis it has become clear that EoE is a food-triggered disease with milk and wheat as the dominant culprit food categories. However, it is still debated whether a disturbed mucosal integrity allowing allergens to cross the mucosal barrier, or changes in wheat and milk manufacturing might induce these inflammatory responses. Furthermore, basic science and clinical studies have accordingly confirmed that a chronic eosinophilic inflammation leads to a remodelling of the oesophagus with micro- and macro-morphological alterations, ending in a strictured oesophagus with impaired function. Fortunately, long-term therapeutic trials, using either topical corticosteroids or dietary allergen avoidance, have demonstrated that this sequela can be prevented or even reversed. This finding is of clinical relevance as it supports the initiation of a consistent anti-inflammatory therapy. Nevertheless, EoE is still an enigmatic disease and the long list of unanswered questions will certainly stimulate further research.

\section{INTRODUCTION}

The identification of a distinct clinicopathological syndrome designated 'eosinophilic oesophagitis' (EoE), with its own clinical and histopathological characteristics, was first described in the early 1990s. ${ }^{12}$ Dysphagia and food impaction are the leading manifestations in adults whereas, in children, food refusal and failure to thrive are the predominant symptoms. ${ }^{3-5}$ Prior to this, descriptions of oesophageal eosinophilia were just single case reports and associated with a variety of disorders, such as gastro-oesophageal reflux, achalasia or eosinophilic gastroenteritis without any specific disease pattern.

Since then, 20 years of intense research by multiple teams have uncovered many molecular, immunological and clinical aspects of this chronic inflammatory disorder. In addition, a close collaboration among many EoE experts has provided a solid base for establishing diagnostic and therapeutic recommendations. ${ }^{3}{ }^{4}$ The publication of the International Consensus Recommendations has been an important milestone in EoE, in particular by defining the disease and impending complications, such as stricture formation and perforation, and by communicating the current treatment modalities. ${ }^{3} 4$ Meanwhile, EoE has reached prevalences comparable with Crohn's disease and is probably the most common cause of solid food dysphagia and of spontaneous perforation of the oesophagus. ${ }^{6}$

In this article we will focus exclusively on recent basic and clinical insights of EoE mainly gathered during the last few years, but we will not provide a systematic overview. As gastroenterologists, we have selected the topics guided by clinical perspectives.

\section{AETIOPATHOGENESIS}

\section{Disturbed mucosal integrity in EoE: chicken or} egg?

Allergen avoidance by dietary measures is successfully used in the treatment of EoE. ${ }^{7-9}$ Based on findings from controlled food reintroduction, milk and wheat have been identified as the leading causative food categories. ${ }^{8-10}$ In westernised countries, milk and wheat have long been staple foods, although apparently EoE only first appeared some 30 years ago. It is thus questionable whether exposure to these two food components actually explains the pathogenesis of EoE. It is more reasonable to assume that a disruption in the mucosal integrity allows milk and wheat proteins to breach the oesophageal epithelium, thereby provoking the mucosal immune system and inducing an inflammatory response. Based on this hypothesis, several researchers have performed morphological, ultrastructural, molecular and functional analyses of the oesophageal barrier, searching for alterations in junctional proteins of desmosomes and of tight junctions.

Rothenberg and colleagues focused on cadherins, a group of junctional proteins within the desmosomes, in particular on the adhesion molecule desmoglein-1 (DSG1). ${ }^{11}$ Using RNA sequencing, quantitative PCR, immunofluorescence, immunohistochemistry, electron microscopic ultrastructural analysis, cell cultures and electrical resistance measurement, the authors searched for morphological and functional consequences of a DSG1 dysregulation in oesophageal tissue from patients with EoE and compared the findings with healthy controls. ${ }^{11}$ This study demonstrates that: (1) DSG1 is more than 20 times downregulated in active EoE compared with controls; (2) DSG1 deficiency of the oesophageal mucosa leads to a structural alteration of the mucosa; and (3) active EoE is associated with a disturbed mucosal integrity. These findings have been confirmed in an independent study showing that, in children with active EoE, the expression of the intercellular junction proteins E-cadherin and claudin-1 is reduced. ${ }^{12}$ This is now accepted as confirmation that, in active EoE, the 
mucosal integrity is altered due to defects in desmosomal and tight junction adhesion proteins. However, the crucial question is whether the impaired barrier function is a non-specific phenomenon evoked by any inflammation in the oesophageal mucosa or, inversely, whether there is inherent barrier dysfunction in EoE that leads to an inflammatory response. Interestingly, it could additionally be demonstrated that the functional defects in desmosomes are reversed by successful treatment of EoE, and that the function of the mucosal barrier of patients with remittent EoE and of healthy controls is almost comparable. ${ }^{11}$ Along with this observation are findings from animal and in vitro studies demonstrating that, in reflux disease, exposure to gastric acid first induces an inflammatory response in the submucosa layer and that mucosal disruption is a secondary phenomenon. ${ }^{13}$

In summary, the finding of an unaltered barrier function in quiescent $\mathrm{EoE}^{11}$ is a solid argument that the disturbed mucosal integrity in EoE is the result-and not the cause-of the chronic eosinophil inflammation. Respecting this interpretation and the fact that milk and wheat are culprit foods for EoE, we have to consider whether changes in wheat or milk manufacturing and processing might lead to allergic responses.

\section{EoE: an environmental disease?}

Dysphagia is the leading symptom in adult EoE. ${ }^{3-5}$ It is well known that mainly the consistency-and not the allergic properties-of the ingested food determines the severity of dysphagia. ${ }^{3-5}$ EoE has different aetiopathogenic pathways from contact dermatitis. Nevertheless, two observations raised the question of whether EoE could be induced by a local interplay between external allergens and the oesophageal surface. First, approximately $80 \%$ of children treated with a protein-free diet achieve a clinicopathological remission. ${ }^{7}$ The efficacy of allergen avoidance by dietary measures has subsequently been repeatedly confirmed and, today, three different types of dietary regimens are used successfully in the treatment of EoE. ${ }^{14}$ The resolution of the eosinophilic inflammation upon allergen avoidance is a strong indicator that a local interaction between ingested allergens and the mucosal immune system of the oesophagus plays a crucial role in the pathogenesis of this condition. Second, one case reporting a close association between pollen load and inflammatory activity of EoE raised the question of whether EoE could be induced by inhaled allergens. ${ }^{15}$ This observation is of particular interest as a link between airway allergen exposure and oesophageal eosinophilia has previously been demonstrated in an animal model. ${ }^{16}$ The response of the oesophageal inflammation to inhaled allergens is therefore a hint that an additional interplay between the mucosal immune systems of the airways and of the oesophagus might be significant in the pathogenesis of EoE. These facts strongly indicate that EoE might be induced by exposure to ingested or inhaled allergens.

Two recently published articles provide additional support for this concept. Miehlke and colleagues reported on an adult female patient undergoing oral immunotherapy for severe pollinosis. ${ }^{17}$ Oral or sublingual immunotherapy is increasingly used to treat patients with IgE-mediated food and pollen allergies, and has the potential to replace the established parenteral immunotherapies. Of note, during this procedure the oral cavity and the oesophagus are repeatedly exposed to high doses of allergens. Four weeks after beginning treatment the patient experienced-for the first time in her life-dysphagia and endoscopically as well as histologically typical signs of EoE. The immunotherapy was interrupted and 4 weeks later the symptoms and signs of EoE disappeared completely. ${ }^{16}$ De novo EoE has also been observed with oral immunotherapy used for the treatment of food allergies in patients with sensitisation and allergy to milk ${ }^{18}$ and eggs. ${ }^{19}$

A case series reported by Dellon and colleagues presented three individuals, two with a history of atopies, who developed EoE after a severe accidental exposure to aeroallergens. ${ }^{20}$ Dysphagia appeared between a few days and 6 months after exposure and subsequently, in all three patients, a de novo onset of EoE could clinically, endoscopically and histologically be confirmed. ${ }^{20}$ Interestingly, the disease did not respond to treatment with swallowed topical corticosteroids, a therapeutic modality normally producing clinicopathological response rates up to $70 \%$ for EoE. ${ }^{20}$ Nevertheless, it might be difficult to distinguish whether an allergen is actually swallowed, inhaled, or even both. The patients developing EoE with oral sensitisation were certainly solely orally exposed. ${ }^{17-19}$ In contrast, the patients reported by Dellon and coworkers obviously had primarily an airway exposure, but swallowing of the allergens probably also occurred. $^{20}$

In conclusion, the results of all these studies show that exposure of the oesophageal mucosa to ingested allergens might induce EoE. Whether an airway exposure can also induce EoE and whether additional paths of induction exist remain to be determined.

\section{Genetic abnormalities in EoE: a sign of predisposition or a sign of disease activity?}

The pathogenesis of EoE is characterised by both a genetic predisposition and an aberrant immune response to environmental antigens. $^{21}$ The first evidence of a genetic predisposition in EoE was provided by reports demonstrating a familial clustering. ${ }^{21-23}$ A positive family history with EoE was found in $7 \%$ of EoE patients in a study of 103 children with EoE. ${ }^{22}$ It is noteworthy that the clinical and pathological features of patients with familial EoE are not different from those of patients with sporadic EoE. ${ }^{24}$ A number of genetic variants have been associated with EoE. Using genome-wide microarray expression analysis on oesophageal tissue biopsies, Blanchard and colleagues found that a single-nucleotide polymorphism (SNP) of the CCL26 gene was associated with susceptibility to EoE. ${ }^{25}$ The association of this SNP with EoE was independent of the atopic status of the individual. ${ }^{25}$ An EoE gene cluster including a defect in filaggrin, a barrier protective molecule that is typically found in the skin, was recently described. ${ }^{25}$ The markedly downregulated filaggrin mRNA levels in patients with EoE compared with control patients might be linked to an oesophageal barrier dysfunction. A genome-wide association study identified a variant on chromosome $5 \mathrm{q} 22$ encompassing the thymic stromal lymphopoietin (TSLP) gene which was strongly associated with EoE. ${ }^{26}$ Polymorphisms in the TSLP have previously been associated with other atopic diseases and increased peripheral blood eosinophil counts. $^{27}$ A genetic variant of the TSLP receptor, which is encoded on a pseudoautosomal region of the $\mathrm{X}$ chromosome, has been linked with EoE in men but not in women. ${ }^{28}$ Recent advances have also been made on a diagnostic level with the development of an 'EoE diagnostic panel' that is based on molecular diagnosis and has demonstrated a high sensitivity and specificity in discriminating paediatric and adult patients with EoE from non-EoE patients. ${ }^{29}$

Clinical features, epidemiological data and genetic evidence suggest that EoE is a polygenic disorder. Distinct SNPs reflect a genetic predisposition, but many molecular abnormalities-for instance, expression of eotaxin-3-is highly variable and correlates with the histological disease activity. ${ }^{25}$ 


\section{NATURAL HISTORY \\ Remodelling in EoE: an inevitable destiny or a preventable risk?}

More than 10 years have passed since the first natural history study demonstrated that EoE is a chronic disease with persistence of symptoms and inflammation over years. ${ }^{30}$ Meanwhile, basic science and clinical studies have accordingly confirmed that a chronic eosinophil-predominant oesophageal inflammation leads to deposition of subepithelial fibrous tissue (figure 1A). ${ }^{3-5}$ This so-called 'remodelling' induces histological, endoscopic, radiological and even functional alterations of the oesophagus, but unfortunately there is currently no standardised method of assessing properly the degree of this process. In contrast, much more is known regarding the molecular, cellular and tissue features of this process. Several cytokines play a role in the oesophageal remodelling. Interleukin 5 (IL-5) has been shown to mediate subepithelial collagen deposition and remodelling processes. ${ }^{31}$ Patients with EoE were found to have an increased expression of fibroblast growth factor 9 and other profibrogenic cytokine genes such as CCL 18 in the subepithelial layer, which suggests that these factors also contribute to the oesophageal remodelling. ${ }^{32} 33$ Oesophageal remodelling has clinical relevance as it is associated with stricture formation (figure 1B) which, in turn, leads to dysphagia and bolus impaction. ${ }^{34}$ Two questions are important regarding this process: (1) is remodelling a process that can be prevented by proper anti-inflammatory treatment; and (2) is subepithelial collagen deposition a one-way process or can an already established fibrosis be reversed by therapeutic interventions?

The first long-term maintenance trial using the topical corticosteroid budesonide has demonstrated that, after 1 year of treatment, the amount of subepithelial fibrosis significantly decreased compared with placebo. ${ }^{36}$ Aceves and colleagues found a significant reduction of oesophageal remodelling in children, even after 3 months of swallowed topical budesonide. ${ }^{37}$ Lucendo and colleagues have confirmed these findings using fluticasone and demonstrated additionally that topical corticosteroid treatment led to a downregulation of profibrogenic cytokine gene expression. ${ }^{33}$ Finally, Chehade and colleagues have demonstrated that, in children, elimination diet reduces subepithelial fibrosis. ${ }^{38}$

In conclusion, long-standing eosinophilic inflammation leads to oesophageal remodelling with subsequent stricture formation. There is strong evidence indicating that swallowed topical corticosteroids as well as dietary allergen avoidance can both prevent and even reverse this oesophageal remodelling process. Remodelling is therefore probably not an inevitable destiny. These findings are of clinical relevance as they support the initiation of a consistent anti-inflammatory therapy.

\section{DIAGNOSTIC TOPICS}

How can we reliably assess symptoms in EoE?

The clinicopathological definition of EoE implies that disease activity should be assessed using a combination of clinical and pathological markers. The clinical part entails patient-reported outcome (PRO) features, particularly symptoms and quality of life aspects. ${ }^{49}$ There currently exists no instrument to measure and assess EoE symptoms that is validated or has the approval of regulatory authorities, but such an instrument is urgently needed to define endpoints for clinical trials and observational studies and also for treating patients with EoE in clinical practice. The current situation poses a challenge for regulatory authorities to approve therapies for EoE management. ${ }^{40-42}$ In an attempt to facilitate the development of clinically meaningful endpoints, the US Food and Drug Administration (FDA) has launched a

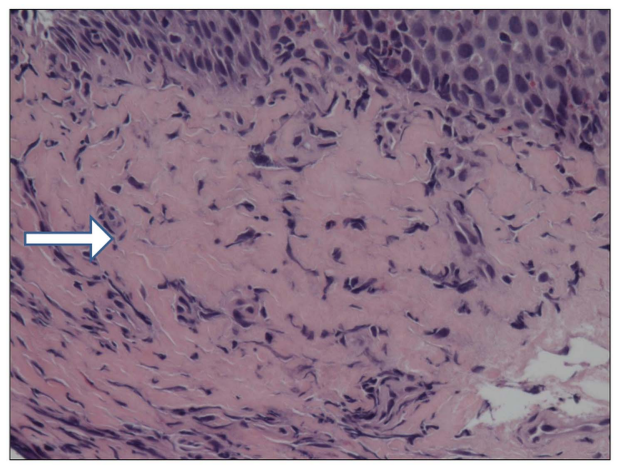

Panel A Oesophageal epithelium from a patient with long-standing Eosinophilic Oesophagitis showing a marked increase of fibrotic tissue in the subepithelial layer (white arrow).

(HE staining, original magnification 400x)

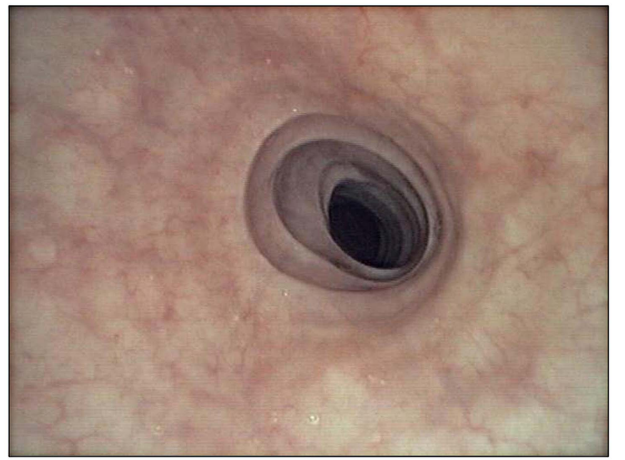

Panel B Endoscopic picture from a patient with Eosinophilic Oesophagitis showing a non-inflamed mucosa but marked rings leading to a severe stenosis of the lumen.

Figure 1 Histological and endoscopic signs of remodelling in eosinophilic oesophagitis. (A) Oesophageal epithelium from a patient with long-standing eosinophilic oesophagitis showing a marked increase of fibrotic tissue in the subepithelial layer (HE staining, original magnification 400x). (B) Endoscopic photograph from a patient with eosinophilic oesophagitis showing a non-inflamed mucosa but marked rings leading to a severe stenosis of the lumen.

collaborative programme that includes patients, physicians, researchers, regulatory authorities and the pharmaceutical industry. $^{41}$

The development and validation of a symptom assessment instrument for paediatric and adult patients with EoE is a challenge for several reasons. First, EoE symptoms typically change in the paediatric population with advancing age. ${ }^{22}$ Second, dysphagia, the leading symptom in EoE, depends on disease-related conditions such as inflammatory activity and stage of fibrosis and also on the consistency of the ingested food and is therefore not easily standardised. Third, symptom severity may also depend on behavioural modification such as food avoidance, food modification or on an altered eating pace. All these factors should be taken into account in the development of an instrument that assesses EoE symptom severity.

So far, three symptom assessment instruments have been developed following guidelines from regulatory authorities. Dellon and colleagues have recently published the 'dysphagia symptom questionnaire' (DSQ), which consists of three questions that are answered on a daily basis and assesses the frequency of dysphagia caused by eating solid food and relief strategies during dysphagia episodes. ${ }^{43}$ The DSQ was evaluated in 35 adolescent and adult EoE patients with clinically and histologically active disease. This 
tool has a 1-day recall period and is easy to use but, so far, has only been field-tested. Further evaluation and validation is required in order to determine stability and responsiveness of this instrument. The international Eosinophilic Esophagitis Activity Index (EEsAI) Study Group is currently developing and validating the PRO instrument that evaluates dysphagia severity according to eight distinct food consistencies and also takes into account behavioural adaptations such as food avoidance, food modification and time to eat a regular meal. ${ }^{39}$ The EEsAI uses a 7-day recall period and assesses dysphagia and also includes consideration of the almost always-occurring avoidance strategies. In 2001, Franciosi and colleagues published a report on the qualitative methods of the Paediatric Eosinophilic Esophagitis Symptom Score (PEESS, V.2.0). ${ }^{44}$

In conclusion, dysphagia does not exclusively depend on the activity of the underlying disorder. The influence of confounders, such as consistency of the ingested food or modification of eating habits, must be considered when dysphagia is used as a marker for disease activity. As noted, several groups are currently developing and validating clinical indices for adult and paediatric EoE.

\section{How can we reliably assess endoscopic abnormalities in EoE?}

In the first publications recognising $\mathrm{EoE}$ as a distinct entity, the endoscopic manifestations were reported as discrete ${ }^{2}$ or even absent. ${ }^{1}$ Subsequently, several endoscopic signs associated with EoE have been identified, such as white spots and coatings mimicking oesophageal candidiasis, ${ }^{45}$ narrowing of the oesophageal lumen, abnormal predisposition for lacerations, ${ }^{46}$ rings giving the oesophagus a trachea-like aspect, longitudinal furrows or invisibility of the capillary vessels. ${ }^{3}$ The better trained that endoscopists are in recognising EoE, the higher the rate of detecting signs of this inflammatory condition. Prospective studies have shown that, today, in more than $90 \%$ of patients with EoE, endoscopic abnormalities can be identified. ${ }^{47}$ The substantial increase in detecting endoscopic signs emphasises the importance of careful and systematic inspection in order to optimise diagnostic capabilities of endoscopy.

EoE can endoscopically be present in an acute inflammatory state, in a state of fibrosis without any active inflammation or as a combination of both. ${ }^{34}$ It is the merit of Hirano and colleagues that a classification and grading system for assessing the endoscopic findings in EoE has been developed. The acronym EREFS designates the five major features of EoE: Exudates (figure 2A), Rings (figure 2B), Oedema (figure 2C), Furrows (figure 2D) and Stricture (figure 2E). ${ }^{48}$ Because familiarity with endoscopic manifestations as well as with its correct terminology is relevant for gastroenterologists, we briefly present this classification and grading system in table 1.

In addition, we have learned that white exudates, furrows and oedema reflect acute inflammation (figure $2 \mathrm{~F}$ ), whereas rings (figure 2B) and strictures (figure 2E) are signs of remodelling. ${ }^{3-5}$

To summarise, today EoE can and should be assessed endoscopically in a structured manner and the EREFS classification is a validated and feasible instrument for performing the assessment.

\section{How can we reliably assess functional properties in EoE?}

According to the consensus recommendations, EoE is defined as a chronic inflammatory disorder of the oesophagus characterised clinically by symptoms reflecting oesophageal dysfunction and histologically by an eosinophil-predominant infiltrate of the oesophageal mucosa. ${ }^{4}$ This definition is based on the fact that
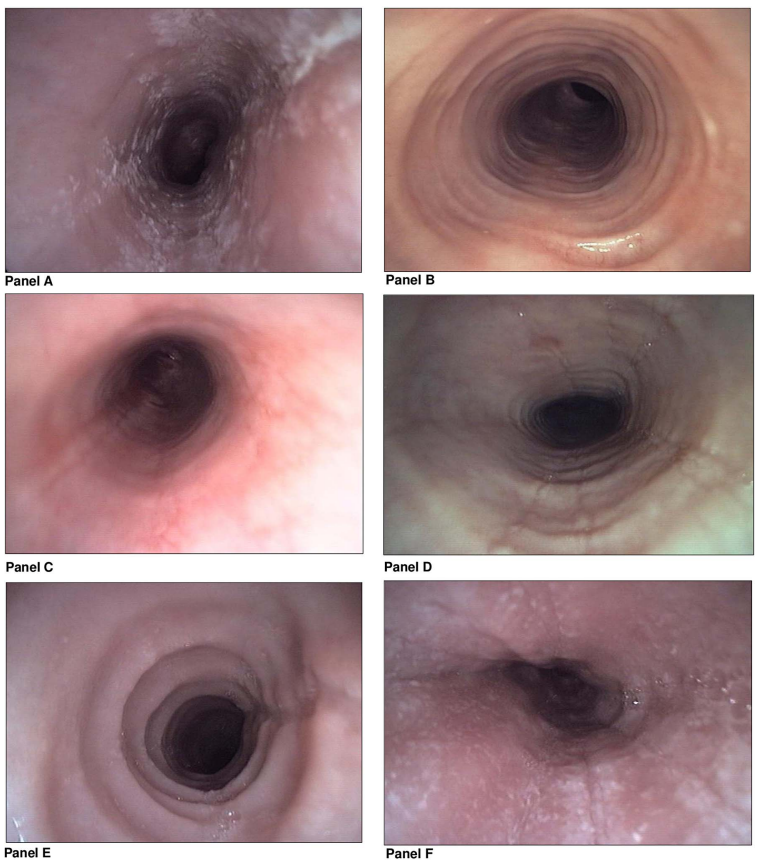

Figure 2 Major endoscopic features of eosinophilic oesophagitis (EREFS classification). ${ }^{48}$ Endoscopic photographs from patients with eosinophilic oesophagitis showing $(A)$ severe white exudates involving more than $10 \%$ of the oesophageal surface; (B) moderate distinct rings not impairing the passage of a standard endoscope; (C) decreased vascular markings and mucosal pallor reflecting mucosal oedema; (D) longitudinal furrows; $(E)$ a severe stricture, also referred to as narrow calibre oesophagus; and (F) a typical combination of several inflammatory signs, in particular exudates, furrows and oedema.

the main function of the oesophagus is to transport a food bolus from the oral cavity to the stomach. Dysphagia and bolus impaction, the leading symptoms in EoE, are therefore typical symptoms of an impaired function of this organ.

Table 1 Major endoscopic features of eosinophilic oesophagitis (EREFS classification)

\begin{tabular}{|c|c|c|}
\hline Endoscopic feature & Grading & Characteristics \\
\hline $\begin{array}{l}\text { White exudates also referred to } \\
\text { as white spots, white plaques }\end{array}$ & $\begin{array}{l}0 \\
1 \\
2\end{array}$ & $\begin{array}{l}\text { Absent } \\
\text { Mild, lesions involving }<10 \% \text { of } \\
\text { the oesophageal surface area } \\
\text { Severe, lesions involving }>10 \% \text { of } \\
\text { the oesophageal surface area }\end{array}$ \\
\hline $\begin{array}{l}\text { Rings also referred to as } \\
\text { concentric rings, corrugated } \\
\text { oesophagus, corrugated rings, } \\
\text { ringed oesophagus, } \\
\text { trachealisation }\end{array}$ & $\begin{array}{l}0 \\
1 \\
2\end{array}$ & $\begin{array}{l}\text { Absent } \\
\text { Mild, subtle circumferential ridges } \\
\text { Moderate, distinct rings that do } \\
\text { not impair passage of a standard } \\
\text { adult endoscope } \\
\text { Severe, distinct rings that do not } \\
\text { permit passage of a standard } \\
\text { adult endoscope }\end{array}$ \\
\hline $\begin{array}{l}\text { Oedema also referred to as } \\
\text { decreased vascular markings, } \\
\text { mucosal pallor }\end{array}$ & $\begin{array}{l}0 \\
1\end{array}$ & $\begin{array}{l}\text { Absent, distinct vascularity } \\
\text { present } \\
\text { Loss of clarity or absence of } \\
\text { vascular markings }\end{array}$ \\
\hline $\begin{array}{l}\text { Furrows also referred to as } \\
\text { vertical lines, longitudinal } \\
\text { furrows }\end{array}$ & $\begin{array}{l}0 \\
1\end{array}$ & $\begin{array}{l}\text { Absent } \\
\text { Present }\end{array}$ \\
\hline $\begin{array}{l}\text { Stricture also referred to as } \\
\text { narrow calibre oesophagus }\end{array}$ & $\begin{array}{l}0 \\
1\end{array}$ & $\begin{array}{l}\text { Absent } \\
\text { Present }\end{array}$ \\
\hline
\end{tabular}


Of note, we have come to recognise that ongoing eosinophilic inflammation leads to so-called remodelling of the oesophagus. ${ }^{3} 4$ Mural thickening, stiffness, increased fragility and friability with an ensuing loss of function are the clinical results of this process. This EoE-inherent feature raises the question as to how one can assess oesophageal function in EoE. This is of particular interest as, so far, the established functional oesophageal tests such as highresolution manometry or impedance/ $\mathrm{pH}$ monitoring have shown equivocal or even conflicting results. ${ }^{3}$

The quantitative assessment of the oesophageal mural compliance using a functional luminal imaging probe (FLIP; EndoFlip, Crospon, Carlsbad, California, USA) is a novel and probably more precise approach for assessing oesophageal function in EoE. The FLIP technology incorporates a multichannel electrical impedance catheter and manometric sensor surrounded by an infinitely compliant bag that is filled with a conducting solution for electrode use. ${ }^{49}$ As the bag is filled with the solution, the probe simultaneously ascertains the oesophageal luminal diameter and pressure at multiple points along the catheter set-up. The resulting pressure-volume curves provide a detailed interrogation of the distensibility of the oesophageal wall. ${ }^{49} \mathrm{~A}$ first study using FLIP in patients with EoE demonstrated a 50\% reduction in the distensibility compared with control subjects. ${ }^{46}$ Nicodème and colleagues recently reported on the assessment of food impaction rates in 70 patients with EoE by highresolution impedance planimetry (FLIP) who were followed prospectively. ${ }^{50}$ The study found that patients with a history of food impactions exhibited significantly lower oesophageal distensibility than those with dysphagia alone. Decreased oesophageal distensibility was also found to be associated with an increased risk of food impaction and need for dilation during the follow-up period. The distensibility plateau was shown to be a more reliable predictor of food impaction risk than findings on endoscopy.

To summarise, the FLIP assesses quantitatively the oesophageal mural compliance, a physiological correlate of remodelling in EoE. This device has therefore the potential to quantify the functional properties in EoE and could be helpful in characterising EoE both at diagnosis and during the follow-up.

\section{PPI-responsive oesophageal eosinophilia: still a mess?}

Since 2009 an increasing number of publications have reported on a group of patients with oesophageal eosinophilia characterised by the following features: (1) a typical EoE symptom presentation; (2) exclusion of gastro-oesophageal reflux disease; and (3) a clinicopathological response to proton pump inhibitors (PPIs). ${ }^{51-55}$ This so-called PPI-responsive oesophageal eosinophilia (PPI-REE) is currently recognised as a disease entity distinct from 'classical' EoE (per definition not responding to PPI), and from gastro-oesophageal reflux disease. ${ }^{56}$

It has been demonstrated independently by Dellon and Moawad that clinical, endoscopic and histological characteristics do not consistently differ between PPI-REE and EoE. ${ }^{57} 58$ Currently, the definition of PPI-REE entails certain flaws as neither 'clinical response' nor 'histological response' is clearly defined. As long as we do not have validated assessment instruments defining clinical, endoscopic and histological activity, we probably cannot expect a clear definition of PPI-REE. To complicate the matter even further, we still lack information about the natural history of PPI-REE. Currently, it seems clear that some patients will develop recurrent symptoms and oesophageal eosinophilia consistent with an EoE diagnosis despite ongoing PPI therapy. ${ }^{59}$ Moreover, the pathogenetic mechanisms leading to an oesophageal eosinophil reduction under PPI are not well understood. PPIs block STAT6, which is involved in binding of the eotaxin-3 promoter in oesophageal epithelial cells. Thus, the PPI response in some patients may be due to an antieosinophil effect of these drugs, independent of their wellknown acid suppressive effect. ${ }^{60}$

In summary, today PPI-responsive EoE is still a poorly understood subset of EoE, a topic of hot debate among experts and indeed a source of uncertainty in the diagnostic investigation of patients with suspected EoE. Clearly more research is needed, especially to refine a PPI-REE diagnosis and to characterise the natural history and pathogenetic mechanisms of this disorder.

\section{Is endoscopy the only procedure to monitor EoE?}

EoE is a chronic condition, ${ }^{30}$ and inherent to chronic diseases is the fact that proper diagnosis is only the first step in management; establishing long-term medical care is equally important. Unfortunately, in EoE the severity of the symptoms does not strongly correlate with the objective degree of inflammation on endoscopy or on histology. ${ }^{61}$ Disease activity thus cannot be reliably assessed based on symptoms alone. ${ }^{50}$ Current standard recommendations that will likely remain valid for the near future therefore dictate that regular follow-up examinations with invasive and costly endoscopic and histological examination remain the gold standard to monitor disease activity. Biomarkers reflecting mucosal inflammation that can be captured in a less invasive fashion are thus urgently needed to follow patients with EoE.

To date, several non-invasive surrogate markers-for instance, determination of eosinophil numbers in the peripheral blood, measuring total IgE levels in the serum or determination of eosinophil-derived specific markers in the faeces or in the blood - have been evaluated. Unfortunately, none showed a sufficient specificity and sensitivity, and their utility could not be recommended as monitoring tools. ${ }^{4}$ However, a promising minimally invasive alternative is the so-called oesophageal string test (EST). ${ }^{62}$ Furuta and colleagues have adapted the Enterotest (HDC Corporation, Pilpitas, California, USA), a technique originally designed to detect gastric and small intestine pathogens, consisting of a capsule and a string. They have evaluated the EST in a total of 41 children with EoE, with reflux disease and in healthy controls. The children had to swallow the capsule and the proximal string was taped to the cheek. After $12 \mathrm{~h}$ the string was removed and, in the luminal effluents, eosinophilderived proteins eluted from the string were measured by ELISA. The results were compared with eosinophil numbers on histology and with content of eosinophil-derived proteins from mucosal biopsies. They found that levels of luminal eosinophilderived proteins-in particular, major basic proteins and Charcot-Leyden crystal proteins-correlated significantly with numbers of oesophageal eosinophils and with levels of the same eosinophil-derived proteins in extracts from oesophageal biopsies. The presence of eosinophil-derived proteins in the luminal secretions therefore reflected the activity of the mucosal inflammation. Of interest, with the hope of simplifying the procedure, preliminary results have shown that a $1 \mathrm{~h}$ exposure of the string might be adequate for monitoring purposes.

Taken together, the EST is a novel minimally invasive technique for measuring oesophageal eosinophilic inflammation and has the potential to facilitate and improve the monitoring of patients with EoE. 


\section{THERAPEUTIC TOPICS}

Medical treatment: when can we expect approval of topical corticosteroids?

In a multitude of studies, swallowed topical corticosteroids (budesonide and fluticasone) have demonstrated efficacy in bringing EoE successfully into clinicopathological remission. ${ }^{3-5}$ It is therefore astonishing that, currently, no drug therapy has been approved by regulatory authorities. There are several reasons for this: (1) performing controlled randomised clinical trials in EoE is still difficult because no instrument for assessing EoE-related symptoms has been approved; (2) some therapeutic studies with swallowed topical steroids have shown a correlation between EoE-related symptoms and histological changes, whereas other trials have documented an isolated histological, but not a symptomatic, response $e^{61}{ }^{63-65}$; the use of various mostly unvalidated symptom assessment instruments might explain these discrepant results; and (3) there is still a debate as to whether the major focus of efficacy should be directed towards symptom relief, anti-inflammatory activity or even both. There is a realistic hope that the intensive and constructive discussions among researchers, pharmaceutical companies and regulatory authorities will soon culminate in a definition of meaningful endpoints. ${ }^{41}{ }^{42}$ Regarding the sequelae of unbridled chronic eosinophilic inflammation such as stricture formation and food impaction, ${ }^{33-35}$ it is likely that patient-reported items as well as biological markers will ultimately be included in the readouts. ${ }^{66}$

Despite all these concerns, there are currently a total of 33 trials evaluating anti-eosinophil medications-swallowed topical corticosteroids and alternative drugs-for EoE registered on the clinicaltrials.gov website. Six of these trials are currently actively recruiting patients. This multitude of trials demonstrates that the search for EoE therapies represents a field of intense research. So far, swallowed topical corticosteroids have proved efficacious in reducing oesophageal eosinophil counts and in improving EoE-related symptoms in several trials, whereas alternative drugs have shown rather limited efficacy. Swallowed topical corticosteroids are therefore currently considered firstline drug therapy. There are not yet any results published on trials comparing fluticasone with budesonide, but both compounds seem to be effective. It is likely that the formulation providing an intensive and long-standing contact between the drug and the oesophageal surface is more relevant than the specific type of corticosteroid. Dellon and colleagues have shown that a viscous form of budesonide was more efficient in reducing eosinophil counts than the nebulised swallowed formulation. In addition, the authors demonstrated a longer oesophageal contact time for the viscous budesonide compared with the nebulised form. ${ }^{67}$ The use of topical steroids is usually safe. Candida oesophagitis has been reported in up to $30 \%$ of patients, with many being noted incidentally during follow-up endoscopy. Long-term safety data regarding potential adrenal suppression and bone mineral density are still awaited.

In conclusion, swallowed topical corticosteroids are highly efficient in treating active EoE and represent the first-line drug therapy for EoE. The challenge is to develop an oesophageal-adjusted formulation. Despite all the above-mentioned hurdles, approval of swallowed topical steroids designed for the treatment of EoE can be expected within the next few years.

\section{Dietary treatment: a reasonable therapeutic modality?}

Since the early days of EoE, allergen avoidance by dietary measures has been an established appropriate treatment. ${ }^{3} 4756$ Today, three dietary modalities are available whose characteristics are illustrated in table 2. The first strategy, elemental diet, consists of a total elimination of all food allergens by an amino acid-based formula. Among all the dietary regimens, this elemental diet represents the most reliable and, ultimately, the most effective strategy for use in children (ie, symptom resolution and histological improvement), with success rates exceeding $80 \% .^{768} 6914$ In adults this approach seems to be less successful, achieving a remission rate of $<50 \%$ in one study. ${ }^{70}$ The second strategy, individually-targeted elimination diets, consists of an elimination of food allergens that have been identified by allergy testing. Targeted elimination diets have the lowest efficacy of all hypoallergic diets, with remission rates between $53 \%$ and $72 \%$ in the paediatric population. ${ }^{1471} 72$ In the adult population the results are even poorer, achieving remission rates between $22 \%$ and $32 \% .{ }^{73}{ }^{74}$ The third strategy, the empirical elimination diet, does not consider the individual allergic status of a patient. It is based on the concept that the empirical avoidance of the food categories that are most commonly associated with immediate hypersensitivity (ie, milk, wheat, eggs, soy, peanuts/nuts, fish/shellfish) would be valuable in treating EoE. After adhering to this so-called six-food elimination diet (6-FED) for 6 weeks, remission rates of more than $70 \%$ were found in children as well as in adults. ${ }^{89}$ Of interest, controlled food reintroduction procedures revealed milk and wheat as the most common triggers for EoE. ${ }^{9} 107576$ Finally, the first longterm study has also shown that an empirical 6-FED was not only able to induce clinical and histological remission of EoE, but also to maintain it up to 3 years. ${ }^{10}$ We can therefore

Table 2 Characteristics of the available dietary modalities for treating eosinophilic oesophagitis

\begin{tabular}{|c|c|c|c|}
\hline \multirow[b]{2}{*}{ Item } & \multicolumn{3}{|l|}{ Type of dietary therapy } \\
\hline & Elemental diet & Targeted elimination diet & Empirical elimination diet \\
\hline $\begin{array}{l}\text { Clinicopathological success } \\
\text { rate }\end{array}$ & $>80 \%$ & $\begin{array}{l}\text { Children } 50-70 \% \\
\text { Adults } 20-30 \%\end{array}$ & $50-70 \%$ \\
\hline Number of eliminated foods & All food groups eliminated & Typically $<6$ foods eliminated & $\leq 6$ foods eliminated \\
\hline $\begin{array}{l}\text { Common food triggers } \\
\text { identified }\end{array}$ & Not applicable & Milk, wheat, egg, soy & Milk, wheat, egg \\
\hline $\begin{array}{l}\text { Number of endoscopies } \\
\text { required }\end{array}$ & $\begin{array}{l}\text { Multiple (one endoscopy per reintroduced } \\
\text { food group) }\end{array}$ & $\begin{array}{l}\text { Multiple (one endoscopy per reintroduced } \\
\text { food group) }\end{array}$ & $\begin{array}{l}\text { Multiple (one endoscopy per reintroduced } \\
\text { food group) }\end{array}$ \\
\hline Drawbacks & $\begin{array}{l}\text { Costly } \\
\text { May require feeding tube } \\
\text { May impact QoL }\end{array}$ & May impact QoL & May impact QoL \\
\hline
\end{tabular}


conclude that all available allergen avoidance approaches have proven efficacy in treating EoE using a non-pharmacological principle.

Regarding these respectable results, one may pose the question as to why the dietary approaches are not more popular. An elemental diet entails the drawbacks of being costly and usually requires a feeding tube. The 6-FED includes several staple foods and therefore requires a fundamental change in nutritional habits. All dietary modalities might have a negative impact on patients' quality of life, and adherence remains an ongoing problem as it has been shown that, as soon as these regimens are no longer followed, EoE returns. ${ }^{9}$ These drawbacks limit the wide use of this regimen.

In order to minimise these obstacles, less restrictive diets have also been evaluated as valuable therapeutic options in treating EoE. In children with a simple milk-free diet, Kagalwalla and colleagues have achieved a remission rate of $65 \%{ }^{76}$ and Lucendo and colleagues have demonstrated that, after identification of the causative food categories by a controlled reintroduction procedure, an individually restricted elimination of the identified causative food is sufficient to maintain EoE in remission. ${ }^{10}$ In one-third of patients only one food category had to be eliminated, in one-third two food categories were involved and, in the last third, three food categories had to be excluded.

Taken together, there is realistic hope that, in the near future, studies evaluating a step-down principle will identify dietary approaches that are easier to follow. This could make diets more popular and provide a valuable therapeutic alternative to swallowed topical steroids in motivated patients desiring a nonpharmacological treatment.

\section{Dilation: still a therapeutic option?}

Oesophageal dilation, either performed using through-the-scope inflatable balloons or wire-guided Savary bougies, represents a valuable therapeutic option for stricturing EoE. It has been clearly demonstrated that unbridled eosinophilic inflammation leads to the formation of oesophageal strictures. ${ }^{3}{ }^{4} 34 \quad 35$ Strictures are, at least in part, responsible for EoE-typical swallowing difficulties and represent the main risk factor for food impactions. ${ }^{34} 77$

As illustrated by several studies, oesophageal dilation can offer longlasting symptom improvement. ${ }^{35} 7879$ A recently reported meta-analysis on 860 patients with EoE, of whom 525 underwent at least one oesophageal dilation and 992 dilations in total, showed a clinical improvement in $75 \%$ of patients. ${ }^{80} \mathrm{In}$ general, patient acceptance of dilation is good $^{78}$; however, dilation has the drawback that it does not influence the underlying eosinophilic inflammation ${ }^{78}$ and that, post-dilation, most patients suffer from marked chest pain for several days. ${ }^{78}$ In addition, in EoE, dilation has the reputation of posing a not insignificant risk of oesophageal perforation. ${ }^{3}$ However, two meta-analyses including 671 and 992 dilations have recently reported perforation rates of $0.1 \%$ and $0.3 \%$, respectively. ${ }^{80} 81$ These rates are comparable with perforation rates for dilation of strictures due to causes other than EoE. In general, considerable symptomatic improvement is achieved when an oesophageal diameter of $16-18 \mathrm{~mm}$ has been reached. ${ }^{78}{ }^{79}$ Nevertheless, in consideration of the inherent EoE oesophageal wall rigidity, it is recommended that the progression steps per dilation session be limited to a maximum of $3 \mathrm{~mm} .{ }^{79}$ Mucosal tears following dilation should not be reported as a complication as they represent a desired therapeutic effect. Post-dilation bleeding necessitating an endoscopic intervention is a rare event. ${ }^{80}$
In conclusion, oesophageal dilation of stricturing EoE is still a valuable therapeutic option and may lead to long-lasting symptom improvement. However, it does not influence the underlying eosinophilic inflammation and, today, the main effort should be towards preventing stricture formation by an early and consistent medical treatment.

\section{FINAL COMMENTS}

Even some 20 years after its first description, EoE remains an enigmatic disease, as illustrated by the discussion of this long list of newly identified basic and clinical aspects. Notably, the list of open questions is even longer and will certainly stimulate further research, which will hopefully soon result in improved care and treatment of patients with EoE.

Acknowledgement We are grateful to Kathleen Bucher for editorial assistance.

Contributors The paper has been written by the two authors in close collaboration.

\section{Competing interests None.}

Provenance and peer review Commissioned; externally peer reviewed.

\section{REFERENCES}

1 Attwood SE, Smyrk TC, Demeester TR, et al. Esophageal eosinophilia with dysphagia. A distinct clinicopathologic syndrome. Dig Dis Sci 1993;38:109-16.

2 Straumann A, Spichtin HP, Bernoulli R, et al. Idiopathic eosinophilic esophagitis: a frequently overlooked disease with typical clinical aspects and discrete endoscopic findings [in German with English abstract]. Schweiz Med Wochenschr 1994; 124:1419-29.

3 Furuta GT, Liacouras C, Collins MH, et al. Eosinophilic esophagitis in children and adults: a systematic review and consensus recommendations for diagnosis and treatment. Gastroenterology 2007;133:1342-63.

4 Liacouras CA, Furuta GT, Hirano I, et al. Eosinophilic esophagitis: updated consensus recommendations for children and adults. J Allergy Clin Immunol 2011; 128:3-20

5 Straumann A, Aceves SS, Blanchard C, et al. Pediatric and adult eosinophilic esophagitis: similarities and differences. Allergy 2012;67:477-90.

6 Hruz P, Straumann A, Bussmann C, et al. Escalating incidence of eosinophilic esophagitis: A 20-year prospective, population-based study in Olten County, Switzerland. J Allergy Clin Immunol 2011;128:1349-50.

7 Kelly KJ, Lazenby AJ, Rowe PC, et al. Eosinophilic esophagitis attributed to gastroesophageal reflux: improvement with an amino acid-based formula. Gastroenterology 1995;109:1503-12.

8 Kagalwalla AF, Sentongo TA, Ritz $S$, et al. Effect of six-food elimination diet on clinical and histologic outcomes in eosinophilic esophagitis. Clin Gastroenterol Hepatol 2006;4:1097-102.

9 Gonsalves N, Yang GY, Doerfler B, et al. Elimination diet effectively treats eosinophilic esophagitis in adults; food reintroduction identifies causative factors. Gastroenterology 2012;142:1451-9.

10 Lucendo AJ, Arias Á, González-Cervera J, et al. Empiric 6-food elimination diet induced and maintained prolonged remission in patients with adult eosinophilic esophagitis: a prospective study on the food cause of the disease. J Allergy Clin Immunol 2013;131:797-804.

11 Sherrill JD, KC K, Wu D, et al. Desmoglein-1 regulates esophageal epithelial barrier function and immune responses in eosinophilic esophagitis. Mucosal Immunol Published Online First: 13 Nov 2013. doi:10.1038/mi.2013.90.

12 Abdulnour-Nakhoul SM, Al-Tawil Y, Gyftopoulos AA, et al. Alterations in junctional proteins, inflammatory mediators and extracellular matrix molecules in eosinophilic esophagitis. Clin Immunol 2013;148:265-78.

13 Souza RF, Huo X, Mittal V, et al. Esophageal reflux might cause esophagitis through a cytokine-mediated mechanism rather than caustic acid injury. Gastroenterology 2009;137:1776-84.

14 Henderson CJ, Abonia JP, King EC, et al. Comparative dietary therapy effectiveness in remission of pediatric eosinophilic esophagitis. J Allergy Clin Immunol 2012;129:1570-8.

15 Fogg MI, Ruchelli E, Spergel JM. Pollen and eosinophilic esophagitis. J Allergy Clin Immunol 2003;112:796-7.

16 Mishra A, Hogan SP, Brandt EB, et al. An etiological role for aeroallergens and eosinophils in experimental esophagitis. J Clin Invest 2001;107:83-90.

17 Miehlke S, Alpan 0, Schröder S, et al. Induction of eosinophilic esophagitis by sublingual pollen immunotherapy. Case Rep Gastroentero/ 2013;7:363-8.

18 Sànchez-Garcia S, Rodriguez del Rio P, Escudero C, et al. Possible eosinophilic esophagitis induced by milk oral immunotherapy. J Allergy Clin Immunol 2012;129:1155-7. 
19 Ridolo E, De Angelis GL, Dall'aglio P. Eosinophilic esophagitis after specific oral tolerance induction for egg protein. Ann Allergy Asthma Immunol 2011;106:73-4.

20 Wolf WA, Jerath MR, Dellon ES. De-novo onset of eosinophilic esophagitis after large volume allergen exposures. J Gastrointestin Liver Dis 2013;22:205-8.

21 Sherrill JD, Rothenberg ME. Genetic dissection of eosinophilic esophagitis provides insight into disease pathogenesis and treatment strategies. J Allergy Clin Immunol 2011:128:23-32.

22 Noel RJ, Putnam PE, Rothenberg ME. Eosinophilic esophagitis. N Engl J Med 2004;351:940-1.

23 Franciosi JP, Tam V, Liacouras CA, et al. A case-control study of sociodemographic and geographic characteristics of 335 children with eosinophilic esophagitis. Clin Gastroenterol Hepatol 2009;7:415-19.

24 Collins MH, Blanchard C, Abonia JP, et al. Clinical, pathologic and molecular characterization of familial eosinophilic esophagitis compared with sporadic cases. Clin Gastroenterol Hepatol 2008;6:621-9.

25 Blanchard C, Wang N, Stringer KF, et al. Eotaxin-3 and a uniquely conserved gene-expression profile in eosinophilic esophagitis. J Clin Invest 2006;116: 536-47.

26 Rothenberg ME, Spergel JM, Sherrill JD, et al. Common variants at $5 q 22$ associate with pediatric eosinophilic esophagitis. Nat Genet 2010;42:289-91.

27 Gudbjartsson DF, Bjornsdottir US, Halapi E, et al. Sequence variants affecting eosinophil numbers associated with asthma and myocardial infarction. Nat Genet 2009:41:342-7.

28 Sherrill JD, Gao PS, Stucke EM, et al. Variants of thymic stromal lymphopoietin and its receptor associated with eosinophilic esophagitis. J Allergy Clin Immunol 2010;126:160-5.

29 Wen T, Stucke EM, Grotjan TM, et al. Molecular diagnosis of eosinophilic esophagitis by gene expression profiling. Gastroenterology 2013;145:1289-99.

30 Straumann A, Spichtin HP, Grize L, et al. Natural history of primary eosinophilic esophagitis: a follow-up of 30 adult patients for up to 11.5 years. Gastroenterology 2003; 125:1660-9.

31 Mishra A, Wang M, Pemmaraju VR, et al. Esophageal remodeling develops as a consequence of tissue specific IL-5-induced eosinophilia. Gastroenterology 2008:134:204-14.

32 Mulder DJ, Pacheco I, Hurlbut DJ, et al. FGF9-induced proliferative response to eosinophilic inflammation in oesophagitis. Gut 2009:58:166-73.

33 Lucendo AJ, Arias A, De Rezende LC, et al. Subepithelial collagen deposition, profibrogenic cytokine gene expression, and changes after prolonged fluticasone propionate treatment in adult eosinophilic esophagitis: a prospective study. J Allergy Clin Immunol 2011;128:1037-46.

34 Schoepfer AM, Safroneeva E, Bussmann C, et al. Delay in diagnosis of eosinophilic esophagitis increases risk for stricture formation in a time-dependent manner. Gastroenterology 2013;145:1230-6.

35 Dellon ES, Kim HP, Sperry SL, et al. A phenotypic analysis shows that eosinophilic esophagitis is a progressive fibrostenotic disease. Gastrointest Endosc 2014;79:577-85.e4.

36 Straumann A, Conus S, Degen L, et al. Long-term budesonide maintenance treatment is partially effective for patients with eosinophilic esophagitis. Clin Gastroenterol Hepatol 2011;9:400-9.

37 Aceves SS, Newbury RO, Chen D, et al. Resolution of remodeling in eosinophilic esophagitis correlates with epithelial response to topical corticosteroids. Allergy 2010;65:109-16.

38 Lieberman JA, Morotti RA, Konstantinou GN, et al. Dietary therapy can reverse esophageal subepithelial fibrosis in patients with eosinophilic esophagitis: a historical cohort. Allergy 2012;67:1299-307.

39 Schoepfer AM. Treatment of eosinophilic esophagitis by dilation. Dig Dis 2014;32:130-33.

40 US Food and Drug Administration. Patient-reported outcome measures: use in medical product development to support labeling claims. http://www.fda.gov/ downloads/Drugs/Guidances/UCM193282.pdf (accessed 3 Dec 2013).

41 Fiorentino R, Liu G, Pariser AR, et al. Cross-sector sponsorship of research in eosinophilic esophagitis: a collaborative model for rational drug development in rare diseases. J Allergy Clin Immunol 2012;130:613-16.

42 Rothenberg ME, Aceves S, Bonis PA, et al. Working with the US Food and Drug Administration: progress and timelines in understanding and treating patients with eosinophilic esophagitis. J Allergy Clin Immunol 2012;130:617-19.

43 Dellon ES, Irani AM, Hill MR, et al. Development and field testing of a novel patient-reported outcome measure of dysphagia in patients with eosinophilic esophagitis. Aliment Pharmcol Ther 2013;38:634-42.

44 Franciosi JP, Hommel KA, DeBrosse CW, et al. Development of a validated patient-reported symptom metric for pediatric eosinophilic esophagitis: qualitative methods. BMC Gastroenterol 2011;11:126.

45 Straumann A, Spichtin HP, Bucher KA, et al. Eosinophilic esophagitis: red on microscopy, white on endoscopy. Digestion 2004;70:109-16.

46 Straumann A, Rossi L, Simon HU, et al. Fragility of the esophageal mucosa: a pathognomonic endoscopic sign of primary eosinophilic esophagitis? Gastrointest Endosc 2003;57:407-12.
47 Kim HP, Vance RB, Shaheen NJ, et al. The prevalence and diagnostic utility of endoscopic features of eosinophilic esophagitis: a meta-analysis. Clin Gastroenterol Hepatol 2012:10:988-96.

48 Hirano I, Moy N, Heckman MG, et al. Endoscopic assessment of the oesophageal features of eosinophilic oesophagitis: validation of a novel classification and grading system. Gut 2013;62:489-95.

49 Kwiatek MA, Hirano I, Kahrilas PJ, et al. Mechanical properties of the esophagus in eosinophilic esophagitis. Gastroenterology 2011;140:82-90.

50 Nicodème F, Hirano I, Chen J, et al. Esophageal distensibility as a measure of disease severity in patients with eosinophilic esophagitis. Clin Gastroenterol Hepatol 2013;11:1101-7.

51 Molina-Infante J, Ferrando-Lamana L, Ripoll C, et al. Esophageal eosinophilic infiltration responds to proton pump inhibition in most adults. Clin Gastroenterol Hepatol 2011:9:110-17.

52 Poh CH, Gasiorowska A, Navarro-Rodriguez T, et al. Upper Gl tract findings in patients with heartburn in whom proton pump inhibitor treatment failed versus those not receiving antireflux treatment. Gastrointest Endosc 2010;71:28-34.

53 Sayei WN, Patel R, Baker RD, et al. Treatment with high-dose proton pump inhibitors helps distinguish eosinophilic esophagitis from noneosinophilic esophagitis. J Pediatr Gastroenterol Nutr 2009;49:393-9.

54 Peterson KA, Thomas KL, Hilden K, et al. Comparison of esomeprazole to aerosolized swallowed fluticasone for eosinophilic esophagitis. Dig Dis Sci 2010;55:1313-19.

55 Moawad FJ, Veerappan GR, Dias JA, et al. Randomized controlled trial comparing aerosolized swallowed fluticasone to esomeprazole for esophageal eosinophilia. Am J Gastroenterol 2013:108:366-72.

56 Dellon ES, Gonsalves N, Hirano I, et al. ACG clinical guideline: evidence based approach to the diagnosis and management of esophageal eosinophilia and eosinophilic esophagitis (EoE). Am J Gastroenterol 2013;108:679-92.

57 Dellon ES, Speck O, Woodward K, et al. Clinical and endoscopic characteristics do not reliably differentiate PPI-responsive esophageal eosinophilia and eosinophilic esophagitis in patients undergoing upper endoscopy: a prospective cohort study. Am J Gastroenterol 2013;108:1854-60.

58 Moawad F, Schoepfer AM, Safroneeva E, et al. Eosinophilic oesophagitis and proton pump inhibitor-responsive oesophageal eosinophilia have similar clinical, endoscopic, and histologic findings. Aliment Pharmacol Ther 2014;39: 603-8.

59 Molina-Infante J, Katzka DA, Gisbert JP. Review article: proton pump inhibitor therapy for suspected eosinophilic esophagitis. Aliment Pharmacol Ther 2013:37:1157-64.

60 Zhang $X$, Cheng $E$, Huo $X$, et al. Omeprazole blocks STAT6 binding to the eotaxin-3 promoter in eosinophilic esophagitis cells. PLoS ONE 2012;7:e50037.

61 Alexander JA, Jung KW, Arora AS, et al. Swallowed fluticasone improves histologic but not symptomatic response of adults with eosinophilic esophagitis. Clin Gastroenterol Hepatol 2012;10:742-9.

62 Furuta GT, Kagalwalla AF, Lee JJ, et al. The oesophageal string test: a novel, minimally invasive method measures mucosal inflammation in eosinophilic oesophagitis. Gut 2013:62:1395-405.

63 Pentiuk S, Putnam PE, Collins MH, et al. Dissociation between symptoms and histological severity in pediatric eosinophilic esophagitis. J Pediatr Gastroenterol Nut 2009:48:152-60.

64 Dohil R, Newbury R, Fox L, et al. Oral viscous budesonide is effective in children with eosinophilic esophagitis in a randomized, placebo-controlled trial. Gastroenterology 2010;139:418-29.

65 Straumann A, Conus S, Degen L, et al. Budesonide is effective in adolescent and adult patients with active eosinophilic esophagitis. Gastroenterology 2010:139:1526-37.

66 Straumann A, Safroneeva E, Schoepfer AM. Placebo and other interventions in asthma. N Engl J Med 2011;365:1446-8.

67 Dellon ES, Sheikh A, Speck 0, et al. Viscous topical is more effective than nebulized steroid therapy for patients with eosinophilic esophagitis. Gastroenterology 2012;143:321-4.

68 Markowitz JE, Spergel JM, Ruchelli E, et al. Elemental diet is an effective treatment for eosinophilic esophagitis in children and adolescents. Am J Gastroenterol 2003;98:777-82.

69 Liacouras CA, Spergel JM, Ruchelli E, et al. Eosinophilic esophagitis: a 10-year experience in 381 children. Clin Gastroenterol Hepatol 2005;3:1198-206.

70 Peterson K, Clayton F, Vinson LA, et al. Utility of an elemental diet in adult eosinophilic esophagitis. Gastroenterology 2011;140(Suppl 1):AB 1080.

71 Spergel JM, Andrews T, Brown-Whitehorn TF, et al. Treatment of eosinophilic esophagitis with specific food elimination diet directed by a combination of skin prick and patch tests. Ann Allergy Asthma Immunol 2005;95:336-43.

72 Spergel JM, Brown-Whitehorn T, Beausoleil JL, et al. Predictive values for skin prick test and atopy patch test for eosinophilic esophagitis. J Allergy Clin Immunol 2007:119:509-11.

73 Molina-Infante J, Martin-Noguerol E, Alvarado-Arenas M, et al. Selective elimination diet based on skin testing has suboptimal efficacy for adult eosinophilic esophagitis. J Allergy Clin Immunol 2012;130:1200-2. 
74 Wolf WA, Jerath MR, Sperry SL, et al. Dietary elimination therapy is an effective option for adults with eosinophilic esophagitis. Clin Gastroenterol Hepatol Published Online First: 17 Jan 2014. doi:10.1016/j.cgh.2013.12.034.

75 Spergel JM, Beausoleil JL, Mascarenhas M, et al. The use of skin prick tests and patch tests to identify causative foods in eosinophilic esophagitis. J Allergy Clin Immunol 2002;109:363-8.

76 Kagalwalla AF, Amsden K, Shah A, et al. Cow's milk elimination: a novel dietary approach to treat eosinophilic esophagitis. J Pediatr Gastroenterol Nutr 2012:55:711-16.

77 Straumann A, Bussmann C, Zuber M, et al. Eosinophilic esophagitis: analysis of food impaction and perforation in 251 adolescent and adult patients. Clin Gastroenterol Hepatol 2008:6:598-600.
78 Schoepfer AM, Gonsalves N, Bussmann C, et al. Esophageal dilation in eosinophilic esophagitis: effectiveness, safety, and impact on the underlying inflammation. Am J Gastroenterol 2010;105:1062-70.

79 Jung KW, Gundersen N, Kopacova J, et al. Occurrence and risk factors for complications after endoscopic dilation in eosinophilic esophagitis. Gastrointest Endosc 2011;73:15-21.

80 Moawad FJ, Cheatham JG, DeZee KJ. Meta-analysis: the safety and efficacy of dilation in eosinophilic esophagitis. Aliment Pharmacol Ther 2013;38: $713-20$.

81 Jacobs JW Jr, Spechler SJ. A systematic review of the risk of perforation during esophageal dilation for patients with eosinophilic esophagitis. Dig Dis Sci 2010:55:1512-15. 